\title{
Operando TEM of Catalysts by In Situ Mass Spectrometry
}

\author{
B.K. Miller and P.A. Crozier \\ School for Engineering of Matter, Transport and Energy, Arizona State University, Tempe, AZ 85287-6106
}

The development of operando techniques has great potential to more fully characterize materials and elucidate mechanisms in catalytic systems. Operando techniques have been successfully adapted from several spectroscopic techniques over the past decade [1]. However, little has been published regarding transmission electron microscopy (TEM) imaging of catalysts with simultaneous activity measurement. Combining TEM and its exceptional spatial resolution with mass spectrometry and its ability to detect small quantities of product gasses from a reaction should allow direct observation of the catalytically active structures in a material.

Partial oxidation of methane is a simple reforming reaction that has been extensively studied [2] for the production of syngas. Ni has also been studied in considerable detail as a catalyst for partial oxidation of $\mathrm{CH}_{4}$ [e.g. 3]. We have therefore chosen to select this catalytic system as the first to study with simultaneous TEM imaging and activity measurement via a mass spectrometer.

To image the catalyst, we use an FEI Tecnai F20 differentially pumped environmental TEM. The mass spectrometer is a Pfeiffer Prisma 80 residual gas analyzer (RGA). The RGA is differentially pumped, since it measures only very low gas pressures. It is also mechanically isolated to reduce its impact on the TEM resolution. Images of the catalyst taken before the partial oxidation reaction are shown in Figure 1. Initial qualitative results from the mass spectrometer attached to the ETEM are shown in Figures 2 and 3. The mass spectra show a transition of the Ni catalyst from a low activity to high activity for $\mathrm{CH}_{4}$ oxidation.

While qualitative interpretation of mass spectra such as those shown in Figure 3 is generally straightforward, quantification of the data from the mass spectrometer is not trivial. Some difficulties arise from the RGA itself, which is not intended to provide quantitative results. Others are inherent in the gas handling system and the gases flowing through it. With operando TEM via EELS, gases of different mass flow through the system differently [4]. In a complex vacuum system, this can result in a different composition reaching the RGA than is present around the sample. Some of these obstacles to quantitative analysis will be described, and the paths toward overcoming them summarized.

\section{References:}

[1] S.J. Tinnemans, et al. Catalysis Today 113, (2006), p.3-15.

[2] C. B. Enger, et al. Applied Catalysis A: General 346, (2008), p.1-27.

[3] S. Chenna, et al. ChemCatChem 3, (2011), p.1051-1059.

[4] Miller and Crozier, ibid (these proceedings)

[5] The support from US Department of Energy (DE-SC0004954) and the use of ETEM at John M. Cowley Center for HR Microscopy at Arizona State University is gratefully acknowledged. 


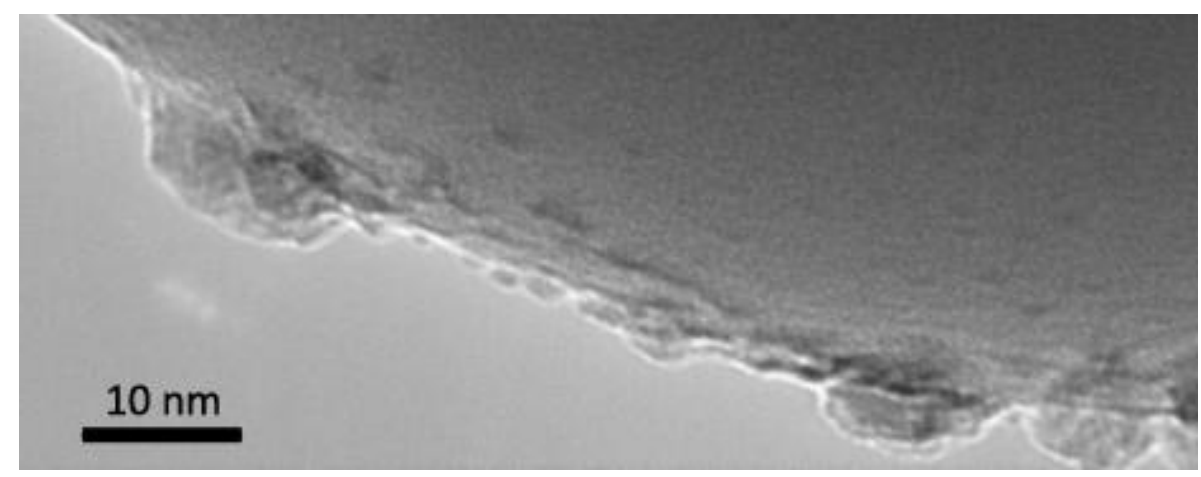

Figure 1. Image of the $\mathrm{Ni}-\mathrm{NiO}$ catalyst supported on $\mathrm{SiO}_{2}$ taken before the reaction began, showing typical Ni-core, NiO-shell structure.

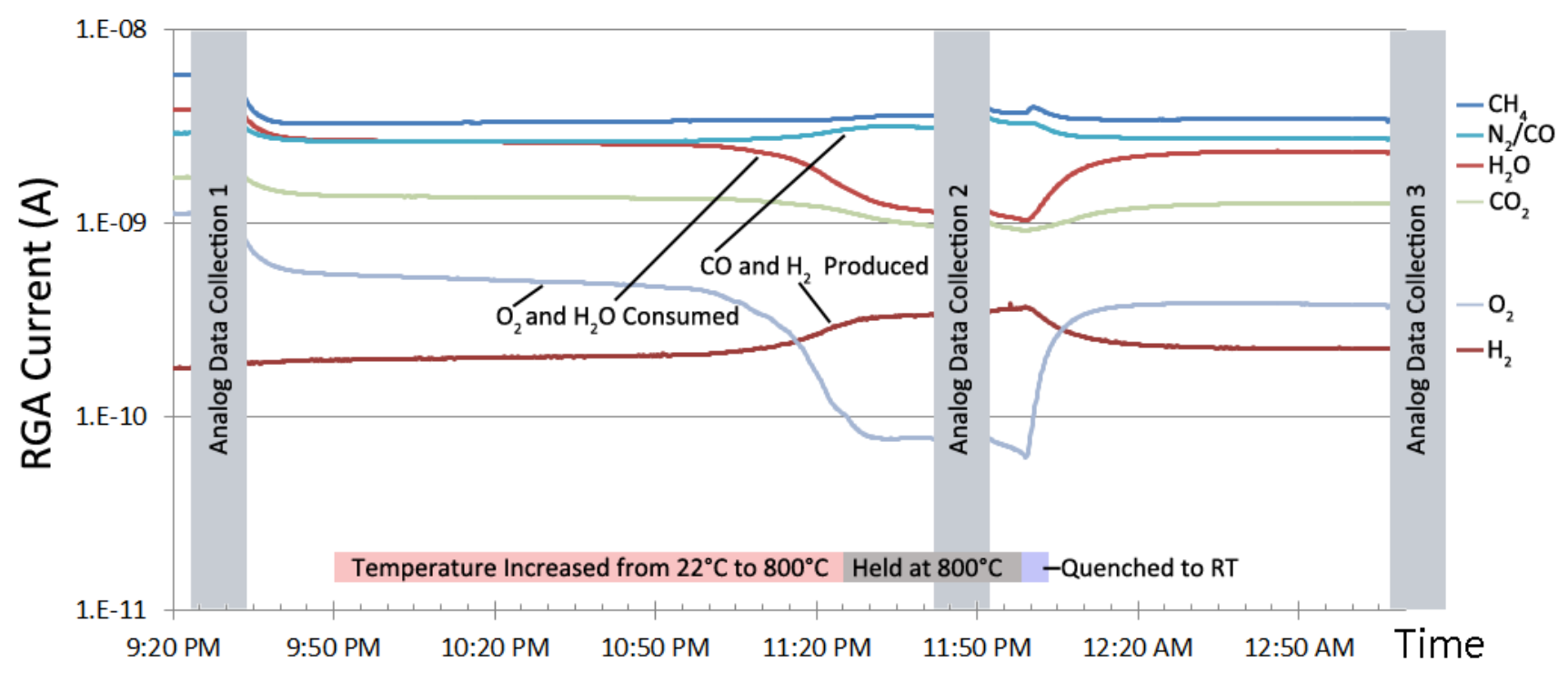

Figure 2. RGA current from select peaks in the mass spectrum corresponding to various relevant gases over time on a $\log$ scale. The consumption of $\mathrm{H}_{2} \mathrm{O}$ and $\mathrm{O}_{2}$ are clear as the temperature approaches $800^{\circ} \mathrm{C}$, as are the production of $\mathrm{H}_{2}$ and $\mathrm{CO}$ over the same period. After the sample is quenched to room temperature, the production drops off, as expected.
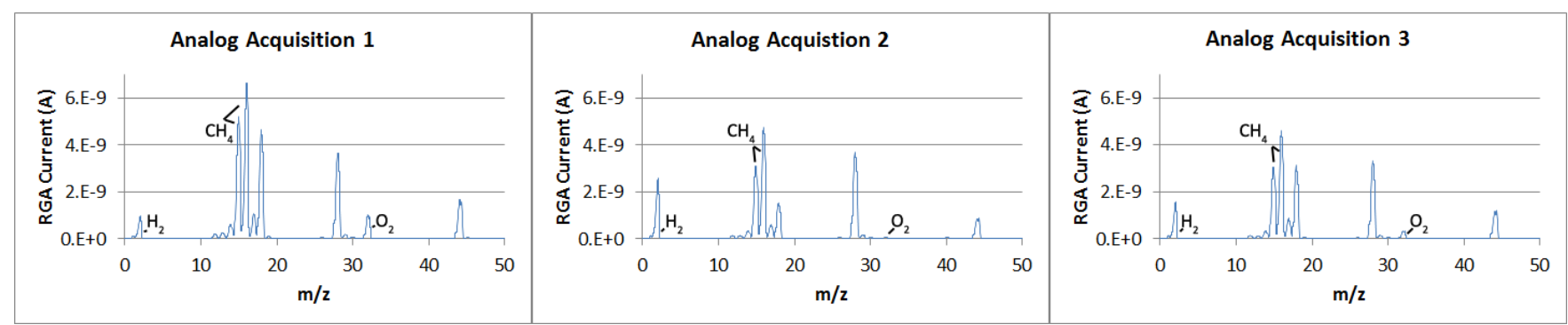

Figure 3. Full analog mass spectra acquired at 3 points in the experiment. Acquisition 1 is taken prior to the reaction, and shows a significant $\mathrm{O}_{2}$ peak, but little $\mathrm{H}_{2}$. Acquisition 2 shows the $\mathrm{O}_{2}$ peak reduced to a background level, while the $\mathrm{H}_{2}$ peak is increased, demonstrating the $\mathrm{H}_{2}$ production. After the sample was quenched, these trends were reversed, as expected. 